\title{
Intracluster correlation coefficients for sample size calculations related to cardiovascular disease prevention and management in primary care practices
}

Jatinderpreet Singh ${ }^{1}$, Clare Liddy ${ }^{1,2^{*}}$, William Hogg ${ }^{1,2}$ and Monica Taljaard ${ }^{3,4}$

\begin{abstract}
Background: Few studies have comprehensively reported intracluster correlation coefficient (ICC) estimates for outcomes collected in primary care settings. Using data from a large primary care study, we aimed to: a) report ICCs for process-of-care and clinical outcome measures related to cardiovascular disease management and prevention, and b) investigate the impact of practice structure and rurality on ICC estimates.

Methods: We used baseline data from the Improved Delivery of Cardiovascular Care (IDOCC) trial to estimate ICC values. Data on 5,140 patients from 84 primary care practices across Eastern Ontario, Canada were collected through chart abstraction. ICC estimates were calculated using an ANOVA approach and were calculated for all patients and separately for patient subgroups defined by condition (i.e., coronary artery disease, diabetes, chronic kidney disease, hypertension, dyslipidemia, and smoking). We compared ICC estimates between practices in which data were collected from a single physician versus those that had multiple participating physicians and between urban versus rural practices.
\end{abstract}

Results: ICC estimates ranged from 0 to 0.173 , with a median of 0.056 . The median ICC estimate for dichotomous process outcomes (0.088) was higher than that for continuous clinical outcomes (0.035). ICC estimates calculated for single physician practices were higher than those for practices with multiple physicians for both process (average 3.9-times higher) and clinical measures (average 1.9-times higher). Urban practices tended to have higher process-of-care ICC estimates than rural practices, particularly for measuring lipid profiles and estimated glomerular filtration rates.

Conclusion: To our knowledge, this is the most comprehensive summary of cardiovascular-related ICCs to be reported from Canadian primary care practices. Differences in ICC estimates based on practice structure and location highlight the importance of understanding the context in which external ICC estimates were determined prior to their use in sample size calculations. Failure to choose appropriate ICC estimates can have substantial implications for the design of a cluster randomized trial.

Keywords: Intracluster correlation coefficient (ICC), Cardiovascular disease, Primary care

\footnotetext{
*Correspondence: cliddy@bruyere.org

'Bruyère Research Institute, C.T. Lamont Primary Health Care Research Centre,

43 Bruyere St (Annex E), Ottawa, ON K1R 7G5, Canada

${ }^{2}$ Department of Family Medicine, University of Ottawa, 43 Bruyere St, Ottawa,

ON K1N 5C8, Canada

Full list of author information is available at the end of the article
}

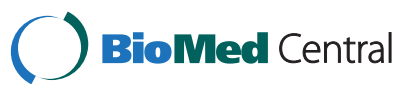

(c) 2015 Singh et al.; licensee BioMed Central. This is an Open Access article distributed under the terms of the Creative Commons Attribution License (http://creativecommons.org/licenses/by/4.0), which permits unrestricted use, distribution, and reproduction in any medium, provided the original work is properly credited. The Creative Commons Public Domain Dedication waiver (http://creativecommons.org/publicdomain/zero/1.0/) applies to the data made available in this article, unless otherwise stated. 


\section{Background}

Cluster randomized trials are increasingly being used in primary health care research [1]. In a cluster randomized trial, groups of individuals (e.g., primary care practices, hospitals, communities), rather than individual patients themselves, are randomly allocated to either an experimental or control intervention. Cluster randomization is required when interventions are necessarily delivered at the group or cluster level, such as an initiative that introduces specialist nurses into primary care practices. In cases where an intervention is delivered on an individual level, cluster randomization may be preferable due to logistical, practical, or scientific reasons [2].

It has become well known that cluster randomized trials are statistically less efficient than trials using individual randomization, as individual responses within a cluster are usually positively correlated [2,3]. The degree of correlation is usually measured by the Intracluster Correlation Coefficient (ICC). To account for intracluster correlation, sample sizes required for cluster randomized trials must be increased to reach the desired power [2]. Sample size calculation formulas for cluster randomized trials are widely available [2,4-6].

One method to account for clustering in sample size estimates, assuming constant cluster sizes, involves using the variance inflation factor (VIF) [2]. The VIF for a cluster randomized trial with a simple parallel design is a function of the cluster size $(\mathrm{m})$ and the intracluster correlation coefficient (ICC) which is denoted by $\rho$ : VIF $=1+(m-1) \rho$. The ICC represents the proportion of variance in a given outcome that can be explained by the variation between clusters, and is given by $\rho=\sigma b 2 /(\sigma b 2+\sigma w 2)$, where $\sigma b 2$ is the between cluster variance and ow 2 is the within cluster variance. An alternative expression for the VIF when the cluster sizes vary is provided by Donner, Birkett and Buck [7].

To conduct sample size calculations for a planned cluster randomized trial, advance estimates of the ICC are required. Estimates are often made available from previously published reports of cluster randomized trials evaluating similar outcomes. Despite recommendations to report ICC values in published reports of cluster randomized trials, [8] it is still challenging to find applicable ICC values to aid in the design of future trials [9].

One concern in using external ICC estimates is whether they are appropriate for the planned cluster randomized trial. If an inaccurate estimate for the ICC is used, the resulting sample size estimate may be either too large or too small. Several studies have analyzed determinants of ICCs [9-12]. Choosing appropriate estimates for ICCs is of particular concern in primary care research, where practice characteristics often vary widely across various domains, including rurality, physician remuneration model, and practice structure (i.e., solo or multiple physician practices).
We recently conducted a large primary care quality improvement initiative in 84 primary care practices across Eastern Ontario, Canada [13]. Through this initiative, we have collected data from 5,140 patients who either have, or are at high risk of developing, cardiovascular disease. The main objective of this paper is to use this rich dataset to: a) report ICC values for a range of process of care and clinical outcome measures related to cardiovascular disease management and prevention, and b) investigate differences in ICC values based on: i) number of physicians per practice (i.e., solo versus multiple physician practices) and ii) urban versus rural practices. Understanding the potential impact that these practice characteristics have on ICC values is important, as a failure to take such factors into account can lead to studies that are inadequately powered.

\section{Methods}

Improved delivery of cardiovascular care (IDOCC) through outreach facilitation trial

The Improved Delivery of Cardiovascular Care (IDOCC) through Outreach Facilitation trial was designed as a stepped wedge cluster randomized control trial to support 84 primary care practices in improving their delivery of evidence-based cardiovascular care for patients at high risk [13]. IDOCC used trained facilitators who worked with practices for 12-24 months to incorporate elements of the chronic care model into daily practice routines to improve secondary preventive care for heart disease, stroke, peripheral vascular disease, chronic kidney disease, and diabetes. Primary care practices were located throughout the Champlain Local Health Integration Network (Ottawa and its surrounding communities) of Ontario, Canada, a culturally diverse region with a population of 1.2 million people who have chronic disease burdens and patient health outcomes that are comparable to Ontario and the rest of Canada. Canada has a publicly funded universal health insurance system, which is often referred to as "Medicare". Detailed information about the recruitment, participants, and data collection can be found elsewhere [13].

In brief, all practices within the Champlain Local Health Integration Network were invited to participate in IDOCC. The Champlain Local Health Integration Network was systematically divided into nine geographic regions, which were grouped together into strata by their location (i.e., west, central, and east). A computer generated randomization approach was used to assign each region within each stratum into one of the three steps of the stepped wedge design. Practices were enrolled in the trial if at least one physician from the practice agreed to participate. In total, 194 physicians in 93 practices were enlisted to participate, with nine practices dropping out prior to the initiation of the study. 
Data were abstracted by chart auditors from a random sample of patient medical charts from participating practices, in order to assess each practice's adherence to evidence-based guidelines for CVD care. Eligible patients for the chart audit were those over 40 years of age who met at least one of the following criteria: (1) CVD including coronary artery disease, cerebrovascular disease (documented stroke and/or transient ischemic attack), or peripheral vascular disease; (2) diabetes mellitus; (3) chronic kidney disease; and/or (4) be at high risk for CVD based on the presence of at least three of the following cardiovascular risk factors: age (males $\geq 45$, females $\geq 55$ ), smoker, hypertension, and dyslipidemia. ICC estimates presented in this paper were calculated from baseline data collected from 5,140 eligible patients (average cluster size of 61 patients/practice; range: 18 to 66). The mean cluster sizes were similar across the 9 regions, ranging from 60 to 65 . In the IDOCC study, patient identifiers were available to uniquely link patients to specific practices, but not to specific physicians within practices. In group practices, it is not uncommon for a patient to be seen by multiple physicians in cases where their primary family physician is unavailable. Also, many group practices have a nurse on staff that performs certain clinical measures (e.g., blood pressure, waistline, weight, etc.) for all patients that are treated in a given practice. As such, inferences were to be made with respect to the practice, rather than individual physicians. For the purpose of presenting ICC estimates, we therefore calculated ICCs within practices. A similar approach was used in several other studies which presented ICC estimates in primary care settings $[9,14,15]$.

Chart auditors collected data related to recommendations from the Champlain Primary Care Cardiovascular Disease Prevention and Management Guideline [16]. Data were collected across four domains: 1) cardiovascular disease/ risk factor screening, 2) drug prescriptions related to CVD, 3) referral to external programs (e.g., referral to smoking cessation program), and 4) clinical test results (e.g., blood pressure readings, lipid profiles, etc.). Process of care data assessed whether recommended care manoeuvres were performed, discussed, or recommended and were recorded as dichotomous indicators, while clinical outcome data were continuous. The Ottawa Hospital Research Ethics Board approved this study (2007292-01H).

\section{Data analysis}

ICC estimates were calculated using an ANOVA approach in which each of the nine geographic regions within the Champlain Local Health Integration Network was treated as fixed strata, corresponding to the study design [17]. ICC estimates were calculated for all patients and separately for patient subgroups defined by condition (i.e., coronary artery disease, diabetes, chronic kidney disease, hypertension, dyslipidemia, and smoking). We compared ICC estimates between practices in which data were collected from a single physician versus those that had multiple participating physicians and between urban versus rural practices. Negative ICC estimates were attributed to sampling error and set to zero [18]. It should be noted that although there are situations that can give rise to true negative ICCs (e.g., when there is competition between clusters), ICCs in the context of cluster randomized trials are generally expected to be positive. ICC estimates may however be negative simply due to chance, particularly when ICC values are close to 0 . SAS 9.3 was used for all analyses.

\section{Results}

Table 1 provides a breakdown of the practice and patient profiles. The 84 participating practices were diverse, varying in practice team structure, physician remuneration approach, and rurality (Table 1 ). Of the 84 practices, 33 were single physician practices, while 51 had multiple physicians on staff (mean: 4, range: 2-19). Of the 51 group practices, 17 only had a single physician consent to participate in

Table 1 Baseline characteristics of practices and patients participating in IDOCC

\begin{tabular}{ll}
\hline Characteristic & $\mathbf{n}(\%)$ \\
\hline Practice Level (N= 84 practices) & \\
Use Electronic Medical Records & $41(48.8 \%)$ \\
Practice structure & \\
Single physician & $33(39.3 \%)$ \\
Multi-Physician Group Practice & $51(60.7 \%)$ \\
Physician remuneration & \\
Fee for service & $45(52.4 \%)$ \\
Capitation & $27(32.9 \%)$ \\
Salary- Community Health Centres & $12(14.6 \%)$ \\
Practice setting & \\
Urban practice & $70(83.3 \%)$ \\
Rural practice & $14(16.7 \%)$ \\
Patient Level (N=5,140 patients) & \\
Age (mean, SD) & $66(12)$ \\
Male (n, \%) & $2,493(48.6 \%)$ \\
Baseline clinical conditions/risk factors & \\
Coronary Artery Disease (n,\%) & $1573(30.6 \%)$ \\
Peripheral Vascular Disease (n,\%) & $327(6.4 \%)$ \\
Stroke/TIA (n, \%) & $666(13.0 \%)$ \\
Diabetes (n, \%) & $2404(46.8 \%)$ \\
Chronic Kidney Disease (n, \%) & $975(19.0 \%)$ \\
Dyslipidemia (n, \%) & $4289(83.6 \%)$ \\
Hypertension ( $\mathrm{n}, \%)$ & $3959(77.1 \%)$ \\
Current Smoker (n, \%) & $1082(21.1 \%)$ \\
\hline & \\
& \\
&
\end{tabular}


IDOCC, and were thus included in the solo physician group for the purposes of this analysis (i.e., 50 solo physicians, 34 multiple physician practices). Patients from such practices had the consenting physician as their main provider; whereas patients from group practices with multiple consenting physicians had different main providers. It is possible that patients in a group practice may be seen by multiple physicians within the same practice, but this likely represents only a small percentage of visits.

ICC estimates obtained from all 84 primary care practices for process of care and clinical outcomes are presented in Tables 2 and 3 respectively. ICC estimates ranged from 0 to 0.173 , with a median of 0.056 (Q1 to Q3, 0.025 to 0.094$)$. The median ICC value for dichotomous process outcomes (0.088) was higher than that for continuous (0.035) clinical outcomes. The largest ICC estimates were for process of care measures looking at waistline measurement (0.173), ACR screening for patients with diabetes (0.167), and two blood pressure readings for patients with chronic kidney disease (0.157).

In general, ICC values were fairly similar across different patient conditions. This may not be surprising as there is substantial overlap between some patient condition subgroups as can be seen from Table 1. For process of care measures (Table 2), medication prescribing across cardiovascular related conditions (i.e., CAD, diabetes, hypertension, and dyslipidemia) tended to have low ICCs (0.01 to 0.04), while ICCs for measuring blood pressure at least twice a year was above 0.1 across all applicable conditions (Table 2). For clinical outcomes (Table 3), all ICC measures were below 0.1, with the exception of measures for diastolic blood pressure for patients with coronary artery disease (0.12) and chronic kidney disease (0.12).

Tables 4 and 5 compare ICC values between practices in which data were collected from a single physician (50 practices) versus multiple physicians (34 practices) for process of care and clinical outcomes, respectively. In general, ICC estimates for process of care measures arising from single physician practices were higher than those arising from multiple-physician practices. ICC values collected from a single physician were on average 3.9 times higher (median: 2.7 times higher) than the value collected from multiple-physician practices, with a maximum difference of 11 times greater for two blood pressure measures per year for patients with diabetes. A similar trend was seen for clinical outcomes as ICC estimates for single physician practices was on average 1.9 times higher (median: 1.8 higher) than multiple physician practices. The largest differences in ICC estimates among all the clinical outcomes were for systolic and diastolic blood pressure measures

Table 6 compares differences in ICC values between urban $(\mathrm{N}=70)$ and rural $(\mathrm{N}=14)$ practices for process of care outcomes. In general, urban practices had higher ICC estimates for process of care indicators, particularly for lipid profile and eGFR measures. In terms of clinical outcomes, ICC estimates were similar between urban and rural practices (results not shown).

\section{Sample calculation}

We now present a hypothetical example to illustrate the potential impact of using inaccurate ICC estimates on the design of a cluster randomized trial. Consider a planned cluster randomized trial aimed at improving the delivery of evidence-based care for high risk patients with hypertension. The primary outcome for the trial is patient systolic blood pressure. The target population consists of solo physician primary care practices. The study is being planned to detect a 5 unit mean difference in systolic blood pressure between intervention and control practices using a two-sided test at the 5\% level of significance with $90 \%$ power, assuming an average cluster size of 20 patients per practice. If the estimated ICC and SD for multiple physician practices were used from Table $5(\mathrm{ICC}=0.020, \mathrm{SD}=16.5, \mathrm{VIF}=1.4)$, the target number of clusters would be 16 practices per arm. On the other hand, if the higher ICC estimate for solo physicians had been used (Table 5: ICC $=0.086, \mathrm{SD}=16.6$, $\mathrm{VIF}=2.6$ ), the sample size requirement would be 31 practices per arm.

\section{Discussion}

To the best of our knowledge, this is the first article to present ICC values for a range of cardiovascular-related outcomes collected from primary care practices in Canada. The ICC values derived from the IDOCC study are in the range of previously reported estimates that have been obtained from primary care settings [1,14,19-21]. In general, ICC values for process of care variables were higher than those for clinical outcomes, which is consistent with findings from other studies $[10,22]$. Intuitively, this is not surprising as clinical outcomes within a given cluster have a greater potential for variability, as each patient will have different levels of compliance and responses to a given treatment.

ICC estimates related to medication prescriptions/recommendations tended to have smaller values than ICCs for other process of care indicators, reflecting little variability amongst practices in prescribing medications for high risk patients. As has been shown in other primary care studies, there is clear evidence and physician agreement on the importance of prescribing medications to high risk patients with cardiovascular disease, diabetes, hypertension and/or dyslipidemia [23-26]. On the other hand, the largest ICC values amongst the process of care indicators were for waistline measurement, two blood pressure screenings per year, and ACR measurement, reflecting relatively high 
Table 2 ICC estimates for process of care outcomes

\begin{tabular}{|c|c|c|c|c|}
\hline Quality of Care Indicator* & $\mathrm{N}$ & Average cluster size & Proportion $^{\dagger}$ & ICC \\
\hline \multicolumn{5}{|l|}{ All patients $(n=5140)$} \\
\hline One blood pressure reading & 5133 & 61 & 0.93 & 0.052 \\
\hline Two blood pressure readings & 5129 & 61 & 0.75 & 0.111 \\
\hline Lipid profile & 5133 & 61 & 0.78 & 0.076 \\
\hline Fasting blood glucose & 5133 & 61 & 0.81 & 0.045 \\
\hline eGFR & 5133 & 61 & 0.80 & 0.066 \\
\hline$A C R$ & 5133 & 61 & 0.36 & 0.122 \\
\hline Smoking status checked & 5132 & 61 & 0.96 & 0.088 \\
\hline Waist circumference & 5140 & 61 & 0.10 & 0.173 \\
\hline \multicolumn{5}{|c|}{ Patients with Hypertension $(n=3959)$} \\
\hline One blood pressure reading & 3959 & 47 & 0.95 & 0.045 \\
\hline Two blood pressure readings & 3957 & 47 & 0.80 & 0.115 \\
\hline Anti-hypertensive medication & 3959 & 47 & 0.94 & 0.015 \\
\hline \multicolumn{5}{|c|}{ Patients with Dyslipidemia $(n=4289)$} \\
\hline Lipid profile & 4289 & 51 & 0.83 & 0.050 \\
\hline Lipid lowering medication & 4289 & 51 & 0.91 & 0.019 \\
\hline \multicolumn{5}{|c|}{ Patients with Diabetes $(n=2404)$} \\
\hline $\mathrm{HbA1c}$ & 2402 & 29 & 0.87 & 0.091 \\
\hline Glycemic Medication & 2402 & 29 & 0.80 & 0.041 \\
\hline 1 blood pressure reading & 2404 & 29 & 0.93 & 0.063 \\
\hline 2 blood pressure reading & 2404 & 29 & 0.76 & 0.133 \\
\hline Lipid Profile & 2404 & 29 & 0.81 & 0.094 \\
\hline Fasting blood glucose & 2404 & 29 & 0.85 & 0.097 \\
\hline eGFR & 2404 & 29 & 0.84 & 0.087 \\
\hline$A C R$ & 2404 & 29 & 0.56 & 0.167 \\
\hline \multicolumn{5}{|c|}{ Patients with Chronic Kidney Disease $(n=975)$} \\
\hline eGFR & 975 & 12 & 0.92 & 0.064 \\
\hline$A C R$ & 975 & 12 & 0.52 & 0.123 \\
\hline 1 blood pressure reading & 975 & 12 & 0.95 & 0.074 \\
\hline 2 blood pressure readings & 974 & 12 & 0.83 & 0.157 \\
\hline \multicolumn{5}{|c|}{ Patients with Coronary Artery Disease $(n=1573)$} \\
\hline Medication & 1573 & 19 & 0.89 & 0.017 \\
\hline 1 blood pressure reading & 1573 & 19 & 0.92 & 0.091 \\
\hline 2 blood pressure readings & 1572 & 19 & 0.77 & 0.137 \\
\hline Lipid profile & 1573 & 19 & 0.75 & 0.104 \\
\hline \multicolumn{5}{|l|}{ Patients who smoke $(n=1082)$} \\
\hline Smoking advice & 1082 & 13 & 0.53 & 0.120 \\
\hline Smoking program & 1082 & 13 & 0.08 & 0.148 \\
\hline Smoking cessation drug & 1082 & 13 & 0.23 & 0.054 \\
\hline
\end{tabular}

${ }^{*}$ whether specified quality of care indicator was discussed, recommended, or performed during a one year timeframe, ${ }^{\dagger}$ represents the proportion of patients that received the listed clinical manoeuvre.

eGFR - Estimated Glomerular Filtration Rate; ACR - Albumin-to-Creatinine ratio; HbA1c - Hemoglobin A1c.

between practice variability for these measures. Unlike medication prescribing for high risk patients, there is likely less agreement amongst physicians regarding the need and/ or appropriateness of doing these process manoeuvres. For example, although the importance of waistline measurement has been widely publicized in predicting all-cause and 
Table 3 ICC estimates for clinical outcomes

\begin{tabular}{|c|c|c|c|c|c|}
\hline Clinical Outcome & $\mathbf{N}$ & Average cluster size & Mean & Standard Deviation & ICC \\
\hline \multicolumn{6}{|l|}{ All patients } \\
\hline SBP & 4771 & 57 & 130.8 & 16.6 & 0.054 \\
\hline DBP & 4771 & 57 & 75.5 & 10.1 & 0.094 \\
\hline LDL & 3805 & 45 & 2.43 & 0.93 & 0.035 \\
\hline $\mathrm{HDL}$ & 3355 & 40 & 3.39 & 0.78 & 0.020 \\
\hline FBG & 4053 & 48 & 6.64 & 2.2 & 0.023 \\
\hline eGFR & 4000 & 48 & 72 & 18.6 & 0.015 \\
\hline$A C R$ & 1589 & 19 & 9.4 & 43.6 & $0.000^{*}$ \\
\hline
\end{tabular}

Patients with Hypertension

$\begin{array}{lll}\text { SBP } & 3746 & 45 \\ \text { DBP } & 3746 & 45\end{array}$

$\begin{array}{lll}132.6 & 16.6 & 0.055 \\ 76.1 & 10.2 & 0.091\end{array}$

Patients with Dyslipidemia

LDL

$3395 \quad 40$

2997

$\mathrm{HDL}$

\section{Patients with Diabetes}

$\begin{array}{ll}\text { SBP } & 2226 \\ \text { LDL } & 1856 \\ \text { HDL } & 1663 \\ \text { HbA1C } & 1999 \\ \text { FBG } & 1986 \\ \text { eGFR } & 1963 \\ \text { ACR } & 1199\end{array}$

Patients with Chronic Kidney Disease

$\begin{array}{lll}\text { eGFR } & 859 & 10 \\ \text { SBP } & 922 & 11 \\ \text { DBP } & 921 & 11\end{array}$

Patients with Coronary Artery Disease

$\begin{array}{ll}\text { SBP } & 1452 \\ \text { DBP } & 1453 \\ \text { LDL } & 1136 \\ \text { HDL } & 1049\end{array}$

Patients who Smoke

\begin{tabular}{ll} 
SBP & 995 \\
DBP & 994 \\
LDL & 757 \\
HDL & 611 \\
\hline
\end{tabular}

SBP - Systolic Blood Pressure, DBP - Diastolic Blood Pressure, LDL - Low Density Lipoprotein, HDL - High Density Lipoprotein, FBG - Fasting Blood Glucose, eGFR Estimated Glomerular Filtration Rate, ACR - Albumin-to-Creatinine ratio, HbA1c - Hemoglobin A1c; *Negative ICCs were considered a result of sampling error and were set to zero.

cardiovascular-related mortality, [27] previous studies have demonstrated that some physicians do not do this screening test due to a lack of time, extra workload and financial implications, while others feel uncomfortable measuring waists or are concerned that patients might get embarrassed [28].
Overall, ICC levels for clinical outcomes were relatively low $(<0.1)$ with the exception of values for diastolic blood pressure. This finding is in line with findings presented by Parker et al. [19], which found diastolic blood pressure measurements to have the largest ICC value from a group of clinical outcome markers 
Table 4 Comparison of ICC estimates for process-of-care indicators between single versus multiple physician practices Quality of Care Indicator*

ICC
Single Physician
$\mathrm{N}=50$

\section{ICC}

Multiple Physicians

$\mathrm{N}=34$

\section{All patients}

One blood pressure reading

0.064

0.154

0.109

0.043

0.066

0.146

0.080

0.237

Waist circumference

Patients with Hypertension

One blood pressure reading

Two blood pressure readings

0.059

0.160

0.019

Anti-hypertensive medication

Patients with Dyslipidemia

Lipid profile

0.069

0.016

Lipid lowering medication

Patients with Diabetes

$\mathrm{HbA1c}$

0.093

glycemic medication

1 blood pressure reading

0.046

0.080

0.192

2 blood pressure readings

0.109

0.082

0.080

0.172

ACR

Patients with Chronic Kidney Disease

eGFR

0.039

0.178

0.089

0.221

2 blood pressure readings

Patients with Coronary Artery Disease

CAD Medication

0.026

0.108

0.205

0.154

0.072

0.136

0.072
0.021

0.029

0.011

0.017

0.041

0.054

0.094

0.091

0.015

0.024

0.011

0.009

0.016

0.037

0.028

0.009

0.017

0.032

0.034

0.059

0.124

0.057

0.033

0.018

0.035

0.009

0.031

0.028

0.018

0.064

0.144

0.034
Ratio

(Single/

Multiple)

3.06

5.24

10.14

2.56

1.61

2.70

0.85

2.59

3.87

6.64

1.63

8.08

1.04

2.51

1.66

8.89

11.42

3.40

2.40

1.35

1.38

0.69

5.35

5.01

6.37

2.95

3.46

7.35

8.67

1.12

0.95

2.11

${ }^{*}$ whether specified quality of care indicator was discussed, recommended, or performed during a one year timeframe. eGFR - Estimated Glomerular Filtration Rate; ACR - Albumin-to-Creatinine ratio; HbA1c - Hemoglobin A1c. 
Table 5 Comparison of ICC clinical outcomes between single physician versus multiple physicians practices

\begin{tabular}{llll}
\hline Clinical & ICC & ICC & $\begin{array}{l}\text { Ratio } \\
\text { Outcome }\end{array}$ \\
& (Single Physician) & (Multiple Physicians) & $\begin{array}{l}\text { (Single/ } \\
\text { Multiple) }\end{array}$ \\
& $\mathrm{N}=50$ & $\mathrm{~N}=34$ & \\
\hline
\end{tabular}

\begin{tabular}{llll}
\hline All patients & & & \\
SBP & 0.082 & 0.020 & 4.10 \\
DBP & 0.133 & 0.051 & 2.60 \\
LDL & 0.042 & 0.029 & 1.44 \\
HDL & 0.024 & 0.024 & 1 \\
FBG & 0.027 & 0.020 & 1.35 \\
egfr & 0.019 & 0.010 & 1.90 \\
acr & $0.000^{*}$ & $0.000^{*}$ & -
\end{tabular}

Patients with Hypertension

$\begin{array}{llll}\text { sbp } & 0.086 & 0.020 & 4.30 \\ \mathrm{dbp} & 0.130 & 0.042 & 3.10\end{array}$

Patients with Dyslipidemia

$\begin{array}{llll}\text { LDL } & 0.044 & 0.023 & 1.91 \\ \text { HDL } & 0.019 & 0.024 & 0.80\end{array}$

Patients with Diabetes

$\begin{array}{llll}\text { SBP } & 0.061 & 0.015 & 4.10 \\ \text { DBP } & 0.108 & 0.053 & 2.04 \\ \text { LDL } & 0.068 & 0.039 & 1.74 \\ \text { HDL } & 0.041 & 0.047 & 0.87 \\ \text { HbA1C } & 0.019 & 0.000 & - \\ \text { FBG } & 0.017 & 0.034 & 0.50 \\ \text { egfr } & 0.016 & 0.018 & 0.89\end{array}$

Patients with Chronic Kidney Disease

$\begin{array}{llll}\text { egfr } & 0.060 & 0.029 & 2.07 \\ \text { SBP } & 0.092 & 0.055 & 1.67 \\ \text { DBP } & 0.131 & 0.078 & 1.68\end{array}$

Patients with Coronary Artery Disease

\begin{tabular}{|c|c|c|c|}
\hline SBP & 0.083 & 0.020 & 4.15 \\
\hline DBP & 0.155 & 0.084 & 1.85 \\
\hline LDL & 0.048 & 0.054 & 0.89 \\
\hline $\mathrm{HDL}$ & $0.000^{*}$ & 0.038 & - \\
\hline \multicolumn{4}{|c|}{ Patients who Smoke } \\
\hline SBP & 0.008 & 0.000 & - \\
\hline DBP & 0.114 & 0.020 & 5.7 \\
\hline
\end{tabular}

*Negative ICCs were considered a result of sampling error and were set to zero SBP - Systolic Blood Pressure; DBP - Diastolic Blood Pressure; LDL - Low Density Lipoprotein; HDL - High Density Lipoprotein; FBG - Fasting Blood Glucose; eGFR - Estimated Glomerular Filtration Rate; ACR - Albumin-to-Creatinine ratio; HbA1c - Hemoglobin A1c.

collected from primary care practices in Rhode Island and Southeastern Massachusetts. The high ICC value likely reflects the hypertension management style of individual physicians [19].
Table 6 Comparison of ICC process of care indicators between urban and rural practices

\begin{tabular}{|c|c|c|c|}
\hline Quality of Care Indicator* & $\begin{array}{l}\text { ICC: Rural } \\
N=14\end{array}$ & $\begin{array}{l}\text { ICC: Urban } \\
N=70\end{array}$ & $\begin{array}{l}\text { Ratio } \\
\text { (Urban/Rural) }\end{array}$ \\
\hline \multicolumn{4}{|l|}{ All patients } \\
\hline One blood pressure reading & 0.056 & 0.052 & 0.92 \\
\hline Two blood pressure readings & 0.119 & 0.105 & 0.88 \\
\hline Lipid profile & 0.012 & 0.082 & 6.91 \\
\hline Fasting blood glucose & 0.021 & 0.047 & 2.22 \\
\hline egfr & 0.016 & 0.075 & 4.83 \\
\hline ACR & 0.133 & 0.115 & 0.86 \\
\hline Smoking status & 0.054 & 0.091 & 1.69 \\
\hline Waist circumference & 0.117 & 0.167 & 1.43 \\
\hline \multicolumn{4}{|l|}{ Hypertension } \\
\hline One blood pressure reading & 0.019 & 0.050 & 2.59 \\
\hline Two blood pressure readings & 0.085 & 0.118 & 1.39 \\
\hline Anti-hypertensive medication & 0.017 & 0.016 & 0.91 \\
\hline \multicolumn{4}{|l|}{ Dyslipidemia } \\
\hline Lipid profile & 0.014 & 0.055 & 4.08 \\
\hline Lipid lowering medication & 0.027 & 0.020 & 0.74 \\
\hline \multicolumn{4}{|l|}{ Diabetes } \\
\hline $\mathrm{HbA1c}$ & 0.026 & 0.090 & 3.48 \\
\hline glycemic medication & 0.027 & 0.045 & 1.68 \\
\hline 1 blood pressure reading & 0.067 & 0.065 & 0.97 \\
\hline 2 blood pressure reading & 0.166 & 0.123 & 0.74 \\
\hline Lipid Profile & 0.014 & 0.105 & 7.68 \\
\hline Fasting blood glucose & 0.057 & 0.097 & 1.69 \\
\hline egfr & 0.021 & 0.097 & 4.56 \\
\hline$A C R$ & 0.142 & 0.164 & 1.16 \\
\hline \multicolumn{4}{|l|}{ Chronic Kidney Disease } \\
\hline egfr & 0.005 & 0.072 & 14.61 \\
\hline$A C R$ & 0.112 & 0.129 & 1.16 \\
\hline 1 blood pressure reading & 0.012 & 0.088 & 7.10 \\
\hline 2 blood pressure reading & 0.063 & 0.177 & 2.82 \\
\hline \multicolumn{4}{|l|}{ Coronary Artery Disease } \\
\hline CAD Medication & 0.029 & 0.016 & 0.53 \\
\hline 1 blood pressure reading & 0.041 & 0.093 & 2.23 \\
\hline 2 blood pressure reading & 0.053 & 0.147 & 2.77 \\
\hline Lipid profile & 0.006 & 0.103 & 15.88 \\
\hline \multicolumn{4}{|l|}{ Smoking } \\
\hline Smoking advice & 0.118 & 0.113 & 0.96 \\
\hline Smoking program & 0.038 & 0.164 & 4.32 \\
\hline Smoking cessation drug & 0.090 & 0.050 & 0.56 \\
\hline
\end{tabular}

*whether specified quality of care indicator was discussed, recommended, or performed during a one year timeframe.

eGFR - Estimated Glomerular Filtration Rate;

ACR - Albumin-to-Creatinine ratio. 
The differences in ICC values between single versus multiple physician practices and those between urban versus rural practices highlight the importance of understanding the context in which ICC estimates are derived before using them in sample size calculations. It can be challenging to find an ICC estimate that was derived from a population and setting that matches the planned study. Researchers may have no choice but to use whatever ICC estimates are available for the outcome of interest, with the assumption that differences in context may only moderately impact sample size estimates. Using an ICC estimate that is too small can result in a substantially underestimated sample size and prevent drawing any definitive conclusions about the results of an intervention. Therefore, it is important to closely examine published ICC estimates to determine whether they are relevant to the planned trial. At the same time, when reporting an ICC estimate, researchers should clearly describe characteristics of the practices included in the trial. Our estimates pertain to the primary care setting; Campbell et al. [10] found that ICCs were significantly higher for secondary care outcomes compared with primary care outcomes.

There were several limitations in this study. Since practices in this analysis consented to take part in the IDOCC study, there is a potential selection bias. Practices that opted to participate in IDOCC are likely more highly motivated and higher performing than provincial averages. As such, it is possible that participating practices have inherent similarities that could decrease between practice variance and result in ICC values that are too small. However, considering that many primary care studies are voluntary, the estimates presented in this paper are likely representative.

In addition, the data for this study were collected from practices across Eastern Ontario only. As such, the results may not be generalizable to primary care studies conducted in jurisdictions with healthcare systems that are very different than in Ontario. However, ICC estimates found in this study were in line with values presented in primary care studies conducted in different countries $[14,19]$.

We have used a simple one-way ANOVA to calculate ICC estimates. This method is commonly used to calculate ICCs for any type of outcome but the resulting value must be interpreted with care if the assumptions of analysis of variance are violated. Eldridge et al. [29] review several alternative definitions of ICCs in cluster randomized trials. Finally, large studies are required to estimate ICCs with a reasonable degree of accuracy. Although our main estimates are based on a relatively large sample size, we have not provided confidence intervals around our estimates. Readers using these ICCs for sample size calculation may therefore need to consider the degree of uncertainty associated with these estimates; in particular, the number of rural practices $(\mathrm{N}=14)$ examined in this study was relatively small. Ukoumunne [30] and Zou and Donner [31] review confidence interval methods for ICCs in CRTs.

\section{Conclusions}

To the best of our knowledge, this article presents the most comprehensive summary of ICC values related to cardiovascular-related outcomes collected from Canadian primary care practices. The ICC estimates presented in this study cover a wide range of conditions and risk factors that can be used to aid in the design of future cluster randomized trials in primary care settings. Furthermore, we observed substantial differences in ICC estimates obtained from single physicians versus a group of physicians; this demonstrates the importance of understanding the context in which ICC values are determined before using them in sample size calculations. Failure to take these differences into account can have substantial implications for the design of a cluster randomized trial.

\section{Abbreviations}

ACR: Albumin-to-creatinine ratio; CAD: Coronary artery disease;

CVD: Cardiovascular disease; eGFR: Estimated glomerular filtration rate;

ICC: Intracluster correlation; IDOCC: Improved Delivery of Cardiovascular Care; SD: Standard deviation; TIA: Transient Ischemic Attack; VIF: Variance inflation factor.

\section{Competing interests}

The authors declare that they have no competing interests.

\section{Authors' contributions}

$\mathrm{BH}, \mathrm{CL}$, and JS conceived the idea for this manuscript. MT and JS conducted the data analysis for this study. All authors critically reviewed and approved the final manuscript.

\section{Acknowledgements}

This project was funded from multiple sources, including the Canadian Institute for Health Research (MOP-1 19508), the Champlain Cardiovascular Disease Prevention Network (CCPN), Pfizer Canada (a founding member of the CCPN), and The Ottawa Hospital Academic Medical Organization's Innovation Fund. The authors would like to acknowledge Liesha Mayo-Bruinsma and Mariam Wiens for helping put this paper together.

\section{Author details}

'Bruyère Research Institute, C.T. Lamont Primary Health Care Research Centre, 43 Bruyere St (Annex E), Ottawa, ON K1R 7G5, Canada. ${ }^{2}$ Department of Family Medicine, University of Ottawa, 43 Bruyere St, Ottawa, ON K1N 5C8, Canada. ${ }^{3}$ Clinical Epidemiology Program, Ottawa Hospital Research Institute, 1053 Carling Ave., Ottawa, ON K1Y 4E9, Canada. ${ }^{4}$ Department of Epidemiology and Community Medicine, University of Ottawa, 451 Smyth Road, Ottawa, ON K1H 8M5, Canada.

Received: 11 November 2014 Accepted: 3 March 2015

Published online: 20 March 2015

\section{References}

1. Eldridge SM, Ashby D, Feder GS, Rudnicka AR, Ukoumunne OC. Lessons for cluster randomized trials in the twenty-first century: a systematic review of trials in primary care. Clin Trials. 2004;1:80-90.

2. Donner A, Klar N. Design and Analysis of Cluster Randomization Trials in Health Research. London: Arnold Publishers; 2000.

3. Cornfield J. Randomization by group: a formal analysis. Am J Epidemiol. 1978;108:100-2. 
4. Campbell MK, Thomson S, Ramsay CR, MacLennan GS, Grimshaw JM. Sample size calculator for cluster randomized trials. Comput Biol Med. 2004;34:113-25.

5. Hayes RJ, Bennett S. Simple sample size calculation for cluster-randomized trials. Int J Epidemiol. 1999;28:319-26.

6. Hemming K, Girling AJ, Sitch AJ, Marsh J, Lilford RJ. Sample size calculations for cluster randomised controlled trials with a fixed number of clusters. BMC Med Res Methodol. 2011;11:102.

7. Donner A, Birkett N, Buck C. Randomization by cluster sample size requirements and analysis. Am J Epidemiol. 1981;114:906-14.

8. Campbell MK, Piaggio G, Elbourne DR, Altman DG. Consort 2010 statement: extension to cluster randomised trials. BMJ. 2012;345:e5661.

9. Adams G, Gulliford MC, Ukoumunne OC, Eldridge S, Chinn S, Campbell MJ. Patterns of intra-cluster correlation from primary care research to inform study design and analysis. J Clin Epidemiol. 2004;57:785-94.

10. Campbell MK, Fayers PM, Grimshaw JM. Determinants of the intracluster correlation coefficient in cluster randomized trials: the case of implementation research. Clin Trials. 2005;2:99-107.

11. Gulliford MC, Adams G, Ukoumunne OC, Latinovic R, Chinn S, Campbell MJ. Intraclass correlation coefficient and outcome prevalence are associated in clustered binary data. J Clin Epidemiol. 2005:58:246-51.

12. Murray DM, Short B. Intraclass correlation among measures related to alcohol use by young adults: estimates, correlates and applications in intervention studies. J Stud Alcohol. 1995;56:681-94.

13. Liddy C, Hogg W, Russell G, Wells G, Armstrong CD, Akbari A, et al. Improved delivery of cardiovascular care (IDOCC) through outreach facilitation: study protocol and implementation details of a cluster randomized controlled trial in primary care. Implement Sci. 2011;6:110.

14. Littenberg B, MacLean CD. Intra-cluster correlation coefficients in adults with diabetes in primary care practices: the Vermont Diabetes Information System field survey. BMC Med Res Methodol. 2006;6:20.

15. Smeeth L, Ng ES. Intraclass correlation coefficients for cluster randomized trials in primary care: data from the MRC Trial of the Assessment and Management of Older People in the Community. Control Clin Trials. 2002;23:409-21.

16. Montoya L, Liddy C, Hogg W, Papadakis S, Dojeiji L, Russell G, et al. Development of the Champlain primary care cardiovascular disease prevention and management guideline: tailoring evidence to community practice. Can Fam Physician. 2011;57:e202-7.

17. Donner A. A review of inference procedures for the intraclass correlation coefficient in the one-way random effects model. Int Stat Rev. 1986;54:67-82.

18. Ukoumunne OC, Gulliford MC, Chinn S, Sterne J, Burney P. Methods for evaluating area-wide and organisation-based interventions in health and health care: a systematic review. Health Technol Assess. 1999;3(5):iii-92.

19. Parker DR, Evangelou E, Eaton CB. Intraclass correlation coefficients for cluster randomized trials in primary care: the cholesterol education and research trial (CEART). Contemp Clin Trials. 2005;26:260-7.

20. Elley CR, Kerse N, Chondros P, Robinson E. Intraclass correlation coefficients from three cluster randomised controlled trials in primary and residential health care. Aust N Z J Pub Health. 2005;29:461-7.

21. Yelland LN, Salter AB, Ryan P, Laurence CO. Adjusted intraclass correlation coefficients for binary data: methods and estimates from a cluster-randomized trial in primary care. Clin Trials. 2011;8:48-58.

22. Taljaard M, Donner A, Villar J, Wojdyla D, Velazco A, Bataglia V, et al. Intracluster correlation coefficients from the 2005 WHO Global Survey on Maternal and Perinatal Health: implications for implementation research. Paediatr Perinat Epidemiol. 2008:22:117-25.

23. Bailey CJ. Metformin: effects on micro and macrovascular complications in type 2 diabetes. Cardiovasc Drugs Ther. 2008;22:215-24

24. Heran BS, Wong MM, Heran IK, Wright JM. Blood pressure lowering efficacy of angiotensin converting enzyme (ACE) inhibitors for primary hypertension. Cochrane Database Syst Rev. 2009:4CD003823.

25. Liddy C, Singh J, Hogg W, Dahrouge S, Deri-Armstrong C, Russell G, et al. Quality of cardiovascular disease care in Ontario, Canada: missed opportunities for prevention - a cross sectional study. BMC Cardiovasc Disord. 2012;12:74.

26. Shepherd J, Blauw GJ, Murphy MB, Bollen EL, Buckley BM, Cobbe SM, et al. Pravastatin in elderly individuals at risk of vascular disease (PROSPER): a randomised controlled trial. Lancet. 2002;360:1623-30.

27. Zhang C, Rexrode KM, van Dam RM, Li TY, Hu FB. Abdominal obesity and the risk of all-cause, cardiovascular, and cancer mortality: sixteen years of follow-up in US women. Circulation. 2008;117:1658-67.
28. Dunkley AJ, Stone MA, Patel N, Davies MJ, Khunti K. Waist circumference measurement: knowledge, attitudes and barriers in patients and practitioners in a multi-ethnic population. Fam Pract. 2009;26:365-71.

29. Eldridge SM, Ukoumunne OC, Carlin JB. The intra-cluster correlation coefficient in cluster randomized trials: a review of definitions. Int Stat Rev. 2009;77:378-94.

30. Ukoumunne OC. A comparison of confidence interval methods for the intraclass correlation coefficient in cluster randomized trials. Stad Med. 2002;21:3757-74.

31. Zou G, Donner A. Confidence interval estimation of the intraclass correlation coefficient for binary outcome data. Biometrics. 2004;60:807-11.

\section{Submit your next manuscript to BioMed Central and take full advantage of:}

- Convenient online submission

- Thorough peer review

- No space constraints or color figure charges

- Immediate publication on acceptance

- Inclusion in PubMed, CAS, Scopus and Google Scholar

- Research which is freely available for redistribution 